\title{
CONSIDERACIONES SOBRE LA EDUCACIÓN EN EL CAPITALISMO GLOBAL CONTEMPORÁNEO
}

\author{
Óscar Julián Cuesta M. ${ }^{58}$
}

\begin{abstract}
RESUMEN
La dinámica contemporánea de producción requiere empleados diferentes a los obreros de la fábrica ideada por Ford. Hoy, la capacidad de trabajo en equipo, de aprender y desaprender rápidamente, de ser creativos y proactivos, son las características que exigen las empresas para sus empleados. Esa nueva forma de producir en el capitalismo contemporáneo, influye en todos los niveles de la educación, pues el sistema necesita sujetos hábiles y competentes, capaces de saber y hacer en el momento y contexto preciso.
\end{abstract}

Precisamente, este escrito reflexiona sobre la educación en el capitalismo contemporáneo, puntualmente, teniendo en cuenta las dinámicas posfordistas y el denominado capitalismo cognitivo. Por último, centra estas reflexiones en la educación a distancia.

Palabras clave: educación, posfordismo, capitalismo cognitivo, educación superior.

\begin{abstract}
Modern production dynamics require employees who are very different from the workers of the factory conceived by Ford. Today, the ability to work in teams, learn and unlearn rapidly, be creative and proactive, are the characteristics that corporations demand of their employees. The new production system of contemporary capitalism influences education at all levels, because it requires skilled and competent individuals who are capable of understanding and executing in the right time and context.
\end{abstract}

To this end, this article reflects on education in contemporary capitalism; specifically, in the light of post-fordian dynamics and so-called cognitive capitalism. Lastly, the article focuses its reflections on distance education.

Key words: education, post-fordianism, cognitive capitalism, higher education.

Recibido: 1 de septiembre de 2009

Aceptado: 23 de octubre de 2009

58 Comunicador social de la Universidad Santo Tomás, especialista en docencia universitaria de la Universidad Cooperativa de Colombia y Técnico en locución y producción de medios audiovisuales del Colegio Superior de Telecomunicaciones. Actualmente, cursa estudios de maestría en educación en la Universidad Pedagógica Nacional. Se ha desempeñado como docente universitario y como educador en proyectos con población vulnerable. 


\section{CONSIDERACIONES SOBRE LA EDUCACIÓN EN EL CAPITALISMO GLOBAL CONTEMPORÁNEO}

\section{INTRODUCCIÓN}

A pesar de que el saber es una fuente de bienestar, el conocimiento siempre ha estado resguardado por unos pocos: desde las prístinas épocas de los primeros hombres, cuando el saber estaba protegido por un chaman, pasando por las grandes abadías donde monjes conservaban con misterio dudas y certezas, hasta los tiempos presentes cuando sólo unos cuantos privilegiados acceden a la educación superior.

Hoy el celo sobre el conocimiento se extiende de manera feroz, no sólo sobre la producción del saber, sino sobre la misma capacidad humana de abstraer la realidad, asignarle un nombre y ponerla en común con sus semejantes: el lenguaje. Tal ferocidad responde a la dinámica de un sistema de producción insaciable que, raudo, superpone sus intereses a la dignidad humana.

Desde el surgimiento del capitalismo el conocimiento ha estado a su servicio. Las primeras máquinas de mediados del siglo XVIII, que respondían a un saber tecnológico novedoso y la organización del trabajo propuesta por Frederick Taylor en los albores del siglo xx, son claro ejemplo de ello.

Para Enzo Rullani (2004, p. 99), "el conocimiento se ha puesto al servicio de la producción” y su "tarea es la de controlar a la naturaleza a través de la técnica y a los hombres a través de la jerarquía". Los resultados para el capitalismo han sido exitosos, pues su influencia se extiende por todos los Estados y se materializa en instituciones transnacionales que rigen la economía mundial.

El conocimiento, que alguna vez la creciente burguesía del Siglo de las Luces le designó un papel emancipador, hoy está subordinado a la producción permanente de la lógica capital. Actualmente, la sociedad forma a sus infantes en aras de continuar con la dinámica capitalista de la producción incesante, de tal forma que las capacidades humanas de la cognición y el afecto hacen parte de la misma producción.

Dicha formación se da con la intención de alcanzar la reproducción de los medios de producción y, en especial, la fuerza de trabajo. Hoy, tal fuerza de trabajo, no sólo responde a la mano, el brazo o el músculo, sino a la creatividad, la capacidad de aprendizaje y las facultades comunicativas propias del hombre, de tal manera que sea calificada para las diversas "exigencias de la división socialtécnica del trabajo, en sus diversos puestos y empleos” (Althusser, 1969).

\section{Formación y productividad}

Antes de la producción capitalista, la formación de la fuerza de trabajo se daba en el mismo taller como lo hacían los aprendices al lado de sus maestros en la Edad Media. En 
el capitalismo, la formación se hace afuera de la fábrica o de la oficina, se da, entre otras instituciones, en la familia y los centros de educación. ¿Cómo se da este proceso?

En el caso concreto del sistema colombiano de educación, el Ministerio de Educación tiene estipulado en los programas de primaria y secundaria el desarrollo de competencias básicas, laborales y ciudadanas en sus educandos. Las primeras hacen referencia al lenguaje, las matemáticas y las ciencias; las segundas, como su nombre lo indica, a las capacidades que permiten cumplir tareas en el plano laboral; y las últimas se fomentan, por ejemplo, con el gobierno escolar o los manuales de convivencia.

Las tres apuntan a generar fuerza de trabajo para el sistema de producción contemporáneo: a tener conocimientos para solucionar inconvenientes en una labor, a ser proactivos, a tener conciencia cívica y profesional, a respetar las órdenes y trabajar en equipo, etc. ${ }^{59}$ Pero esa calidad de la mano de obra no implica solamente tales habilidades, pues, en palabras de Althusser (1969), conlleva "sumisión a las reglas del orden establecido". Para ello, según este autor, el Estado permite que entes privados (la familia, el sistema escolar, la religión, los medios de comunicación, el aparato político y cultural), cumplan su papel como aparatos ideológicos, ejerciendo presión simbólica para que se mantengan las "relaciones de producción que son, en últimas, relaciones de explotación”. En pocas palabras, se trata de que la clase dominada siga sumisa a la clase dominante, lógica intrínseca al capitalismo en sus diferentes etapas:

"La reproducción de las relaciones de producción, objetivo último de la clase dominante, no puede ser una simple operación técnica de formación y distribución de los individuos en los diferentes puestos de la 'división técnica' del trabajo: toda división 'técnica', toda organización 'técnica' del trabajo es la forma y la máscara de una división y una organización social (de clase) del trabajo" (Althusser, 1969).

\section{Posfordismo}

La formación de los futuros sujetos que ingresaran al mercado laboral es una preocupación fundamental del sistema capitalista, de allí la importancia de diseñar programas curriculares y estructurar docentes para consolidar sus diferentes intereses. En las últimas décadas, dichos intereses responden a las cualidades de una etapa particular: la etapa posfordista.

Como lo indica su título, el posfordismo es la dinámica posterior al modelo fordista de producción; este último, estaba basado en una jerarquía y en una marcada cadena de mando, de tal manera que el saber y las decisiones están en la potestad de los directivos y cada empleado se limita a conocer su labor, que está previamente establecida en la cadena de montaje.

59 Ver la Serie Guías N. 21, del Ministerio de Educación Nacional, titulada Articulación de la educación con el mundo productivo. 
Por su parte, el posfordismo, que surge para mejorar los niveles de producción y los inconvenientes propios del anterior modelo a partir de una participación activa de los trabajadores, se basa en una estructura menos rígida y en la cual los empleados conocen la totalidad del proceso. Es una forma de producción articulada en equipos de trabajo y de manera flexible. Para evitar llenar las bodegas de productos, se produce de acuerdo a la demanda. Promueve la comunicación, la toma decisiones y la capacidad de estar en constante aprendizaje, de estar a la par con la tecnología.

Aunque es importante aclarar que fordismo y posfordismo tienen vigencia hoy en la producción dependiendo el renglón económico, la educación tiende a formar sujetos para satisfacer las necesidades posfordistas, como se puede ver en las competencias citadas anteriormente, pues se necesita de empleados proactivos, a los que no se "tenga que arriar, sino atajar", a personas creativas capaces de solucionar problemas en la misma coyuntura y con una constante capacidad comunicativa.

Como lo afirma Giuseppe Cocco y Carlo Vercellone, esto ocurre porque hoy la producción responde a otras necesidades más sofisticadas, a un consumo complejo, diferente al de principios del Siglo xx. Además, el posfordismo responde a un modelo propio de las revoluciones tecnológicas y a "las nuevas características de las figuras obreras nacidas de la revolución informática".

\section{Capitalismo cognitivo}

Profundizando en este planteamiento, para Moncayo (2007), la producción contemporánea va más allá del posfordismo y recibe el nombre de capitalismo cognitivo, el cual tiene un gran rasgo peculiar: la información. "La información es el nuevo objeto principal del trabajo", esto evidenciado en el manejo de hardware y software, que propicia la "des-localización o desterritorialización y la globalización de la producción” (p. 27).

Asimismo, para Moncayo (2007) el capitalismo cognitivo va más allá de la subordinación del trabajo vivo al sistema de máquinas, que eran la materialización del intelecto general, éste se hace presente en los sujetos, dando lugar a lo que se ha denominado trabajo inmaterial. Lazzarato (2001) ubica este tipo de trabajo en el contexto de la fábrica difusa, el trabajo descentralizado y las formas de tercerización, lo cual implicó otro tipo de individuo para llevar a cabo la producción. Dicho tipo de trabajo recibe el título de inmaterial porque es intangible, difuso y disperso, marcado por una constante comunicativa y cooperativa.

Los instrumentos con los cuales se realiza el trabajo es el cerebro y la dimensión afectiva (emocional) de los sujetos, lo cual marca una determinante diferencia con las primeras etapas del capitalismo: antes el capitalista poseía los instrumentos (máquinas) y el obrero actuaba en función de ellas. Hoy el sujeto ya no recibe un salario por el alquiler de su fuerza de trabajo, ahora está marcado por otras formas, como lo referencia Maurizio Lazzarato (2004), quien piensa que "el trabajo artístico tiende a convertirse en uno de los modelos de la producción de la riqueza" (p. 139). 
Desde su perspectiva, "La cultura ha dejado de ser un refinanciamiento de la burguesía para pasar a ser uno de los motores del capitalismo contemporáneo con nuevas formas de organización del trabajo y, justamente, las formas de organización del trabajo en la producción cultural están a la cabeza de la organización capitalista" (Lazzarato, 2007, p. 145). Como ejemplo de ello se puede traer a colación la flexibilidad de los contratos por temporadas, pues la mayoría de profesionales no son empleados fijos de una empresa, sino tienen trabajos intermitentes dependiendo de la coyuntura de la producción.

Las nuevas maneras de producción, donde corre raudo el trabajo inmaterial, han modificado las formas en que se reproducen los medios de producción, en especial, lógicamente, las fuerzas de trabajo. Esto se puede ver reflejado en las reformas a la educación, tanto básica como media y superior. Fundamentalmente, se deja de lado la formación de los saberes rígidamente especializados, para desarrollar los rasgos generales, como lo son las competencias lingüísticas, pues, según Virno (2003), el capitalismo contemporáneo tiene su principal recurso en las capacidades comunicativas del ser humano.

La fuerza de trabajo, es decir, las capacidades psíquicas, físicas y cognitivas del obrero, están hoy sujetas a la demanda laboral de manera completa y constante, pues ya el trabajo especializado, que privilegiaba alguna de las capacidades, ha sufrido una gran transformación. El mundo contemporáneo asiste en este momento a la despiadada sumisión de la complejidad humana al interés avaro del sistema, ya no se trata de repetir una operación en una fábrica, se trata de ser un obrero 'integral', que descansa y se recrea en función de la empresa.

Sumado a lo anterior, la fuerza de trabajo en el marco del capitalismo cognitivo está condenada a ser obsoleta rápidamente, de allí el menesteroso afán de capacitarse constantemente y ser flexibles en sus capacidades laborales, de resolver tareas que no sean de su oficio o brindar soluciones a problemas que se presenten en una coyuntura dada. Además, deben estar en la capacidad de manejar el caudal de información disponible y fluctuante, con el ánimo de crear e innovar. (Moncayo, 2007).

\section{Educación superior}

Para empezar la discusión en el marco de la educación superior, la reproducción de la fuerza de trabajo está siendo formada bajo parámetros que son aconsejados, si cabe el eufemismo, por instituciones económicas de corte transnacional, lo cual permite deducir de manera explícita su intención mercantil en defensa de los intereses del sistema contemporáneo de producción.

Anteriormente, la universidad cumplía con el papel de producir profesionales en campos específicos y disciplinas concretas, satisfaciendo el pensamiento positivista propio del capitalismo del siglo pasado. Sin embargo, gracias a las tendencias críticas de corrientes teóricas como la expuesta por la Escuela de Frankfurt, las instituciones de educación superior cumplieron un papel más reflexivo, analizando problemas sociales propios de la inicua dinámica del sistema. "Por ello la universidad, en especial la pública, era estigmatizada bajo el signo político; los ataques contra ella provenían (...) desde la perspectiva de cerrar, incluso 
por medios represivos directos, espacios que permitían la libre circulación del pensamiento político y social" (Moncayo, 2007, p. 36).

Aún más, el espacio de la educación superior dejó de ser un camino que abre las puertas al asenso social, que permitía la "igualdad de oportunidades" (Múnera, 2007 p. 14), para convertirse en un escenario que excluye y continúa perpetuando a una minoría dominante y una masa sumisa: como lo platea Moncayo (2007), a las universidades privadas sólo accede un porcentaje de privilegiados y a las públicas dejan de acceder miles que se rezagan por la ausencia de cobertura.

Esta poca cobertura en la educación superior, obliga a los educandos a buscar institutos técnicos y tecnológicos, creando más operarios calificados que investigadores y productores de nuevos saberes, más fuerza de trabajo capacitada que individuos con actitud crítica y reflexiva. Igualmente, va perpetuando una pequeña elite que sí accede a saberes más complejos, como son los programas de posgrado.

Deliberadamente la educación superior está siendo dirigida a satisfacer las necesidades del mercado, de tal manera que la oferta de los programas y las propuestas curriculares se orienta en "función de la observación de los requerimientos del sector productivo" (Moncayo, 2007, p. 47), evidenciando la imposibilidad de construir saberes ajenos a la maquinaria capitalista y, mucho menos, alternativas diferentes a las formas del sistema.

Ese cause por el cual corre la educación es un sendero planificado en detalle y su discurso para los oídos incautos es llamativo y convincente: se habla de competencias, de calidad, pertinencia y relevancia, lo cual, a primera vista, es razonable, pero es menester alcanzar otros niveles de calidad, los programas deben responder al contexto y ser pertinentes a la realidad de la comunidad, etc. Sin embargo, no mencionan que la calidad no significa educandos gomosos del saber, buscadores del conocimiento, sino mano de obra calificada, o que la pertinencia responde a las prioridades del modo de producción y no a las dificultades sociales propias de una nación históricamente injusta.

En aras de cercenar de tajo la generación de alumnos con otra visión reflexiva, arguyen que "ante la dinámica contemporánea del conocimiento, que lo ha convertido cada vez más provisional e inestable” (Moncayo, 2007, p. 49), no es preciso centrar la educación en la apropiación de conocimientos, de tal manera que no es importante saber sino saber hacer con lo que se sabe. En ese orden de ideas, la formación gira entorno a los instrumentos que permiten el saber y no en el conocimiento mismo. 'Aprender a aprender' sentencia la directriz, pues es vital que en el modelo del trabajo inmaterial, el funcionario resuelva el problema sin la dilación de esperar la decisión de las cabezas de la jerarquía.

Henry Ford, industrial que transformó la forma de producción capitalista, de allí el término fordismo, dijo alguna vez que "pensar es el trabajo más difícil que existe. Quizás sea esta la razón por la que haya pocas personas que lo practiquen". Los paladines del capitalismo contemporáneo, para asegurar que nadie ejecute ese trabajo forzoso, han delimitado las 
funciones de las instituciones educativas a aparatos ideológicos que reproducen los medios de producción, en su caso, fuerza de trabajo sin espíritu de oposición.

Para cumplir a cabalidad la formación de educandos en la dinámica contemporánea de producción, el sistema educativo está ofreciendo dos modalidades según los parámetros del mercado. Estas son las empresas transnacionales de educación y las universidades corporativas; las primeras responden a intereses lucrativos que se alcanzan a través de un mayor número de matriculas pagas, las segundas, no muy diferentes a la esencia de las otras, responden a universidades de corporación propiamente dichas, donde se forman los profesionales conforme a lo que necesita x o y empresa, de tal manera que se incremente "la eficiencia de la fuerza laboral mediante una mejor adaptación práctico-cognitiva al proceso productivo". (Múnera, 2007, p. 12)

Ver la educación como un reglón de la economía de servicios desmitifica el papel que tuvieron las instituciones de educación formal. En el caso de la universidad, otrora espacio de expresión cultural, de aljibe del saber, de análisis de las dinámicas sociales, ha perdido su esplendor gracias la "banalización del sentido del saber académico y científico en las sociedades contemporáneas" (Múnera, 2007, p. 13). Banalización que, como se ha dicho a lo largo de este escrito, tiene la deliberada intención de reproducir individuos sin el gozo por el prurito intelectual, sino por la apropiación de unas competencias generales que permitan el trabajo en equipo, el cumplimiento de horarios y metas y la comunicación pertinente, para lo cual refuerza la consolidación de un intelecto general, directamente productivo, materializado en los lugares comunes, formas lingüísticas lejanas a los espacios culturales específicos donde son generados los conocimientos del conocimiento (Virno, 2003).

\section{Reflexiones finales}

Al principio de este artículo se afirmó que el conocimiento ha estado siempre en manos de unos cuantos, minoría que, recordando la alegoría platónica, ha guiado a una mayoría a través de las sombras de la caverna, pues nunca le han prestado la luz para observar directamente y con claridad. A raíz de las reformas a la educación en sus diferentes niveles, se evidencia que la gran mayoría seguirá confinada a ver las sombras desde la caverna, pues sólo unos pocos están destinados a ser productivos por encima de todo, incluso, con la complejidad misma de su facultad cognitiva y lingüística.

En ese orden de ideas, es inminente la resistencia. En un principio, a partir de la denuncia pública de esta deliberada reproducción de la fuerza de trabajo al modo posfordista y luego satisfaciendo las exigencias propias del capitalismo cognitivo. Es menester rescatar la dignidad humana, la complejidad propia del saber humano, del mismo conocimiento, de la potestad que tiene cada individuo sobre sí mismo, sobre su ser político, teórico y social, para reivindicarlo de la sumisión reiterada al sistema. Es hora de poner coto al hombre destinado, ciegamente, a producir para consumir. 
La coyuntura misma de la educación, presenta la posibilidad misma de restablecer la resistencia y motivar el cambio. El conocimiento permite la libertad y, en consecuencia, la educación podría permitir la emancipación. La reflexión, aún vigente en algunos espacios académicos, debe observar de manera constante los procesos que se están presentando en la producción capitalista contemporánea y su consecuente influencia en la superestructura social.

La misma educación permite formular opciones a la presente coyuntura capitalista: "El reto, por lo tanto, no es sólo que tendencias dominantes y las dificultades del proceso en curso sean analizados y criticados más allá de los lugares comunes y de los entendimientos tradicionales, sino lograr que los espacios de la educación superior y de la universidad sigan siendo o vuelvan a ser escenarios para la confrontación de las políticas estatales, planteando orientaciones alternativas" (Moncayo, 2007, p. 52).

Precisamente, ahora que los estados han tenido que revalidar su papel debido a la crisis mundial de los mercados, producto de la avaricia de los banqueros agiotistas, es propicio resignificar el papel del Estado en la formación de los ciudadanos, en la educación de las personas. Ya que el neoliberalismo a ultranza está cuestionado, revalidar el papel de los estados es una tarea que no admite dilación, pues su discusión es vital para el bienestar de las comunidades. Esa coyuntura es la oportunidad propicia para revalidar el papel de las universidades y reivindicar el conocimiento y la reflexión académica.

\section{Sobre la educación a distancia}

Para finalizar, es pertinente motivar la investigación y la reflexión sobre el papel de la educación a distancia en la formación de sujetos en el capitalismo contemporáneo, dado que para muchas personas este tipo de educación es la única forma de acceder a procesos formales de profesionalización, supliendo las necesidades de un gran grupo de ciudadanos que por costos, disponibilidad de tiempo y dificultades en su desplazamiento optan por esta opción.

El bombardeo publicitario de entidades que ofrecen programas a distancia ha tenido un notorio aumento en el último lustro, ofreciendo titulaciones rápidas y a bajo costo. El afán de obtener títulos para mejorar las opciones laborales ha llevado a que lo importante sea "el cartón" y no el saber, veleidad contemporánea a la cual hay que poner coto, pues las sociedades, como por ejemplo la colombiana, exigen investigadores de hecho y no de nombre.

Sin duda, en este panorama, es importante preguntarse sobre la calidad de tales programas, dado que la educación a distancia exige, por sus particulares dinámicas, una metodología que garantice el proceso de enseñanza-aprendizaje, para que la formación como profesional sea coherente con las expectativas y necesidades de los educandos.

Puntualmente, es importante reiterar que el programa de toda universidad a distancia debe propender por la formación de sujetos críticos y comprometidos con los problemas sociales que acaecen a su comunidad, de allí la obligación de educar a nuevos investigadores y no, como se dijo a lo largo de este escrito, más manos para el sistema. 


\section{REFERENCIAS BIBLIOGRÁFICAS}

ALTHUSSER, LOUIS. (1969). Ideología y aparatos ideológicos del Estado. Recuperado el 10 de septiembre de 2008 de http://www.ucm.es/info/eurotheo/e_books/althusser/index.html.

COCCO, GIUSEPPE Y VERCELLONE, CARLO. (S.F). Los paradigmas sociales del posfordismo. Recuperado el 21 de noviembre de 2008 de http://rcci.net/globalizacion/2002/ fg271.htm

FORD, HENRY. (----). El libro de las citas. Las mejores frases de la historia. (Se desconoce ciudad): Revista Muy Interesante.

LAZZARATO, MAURIZIO. (2001). Trabajo inmaterial. Río de Janeiro: DP\&A editora.

LAZZARATO, MAURIZIO. (2004). Tradición cultural europea y nuevas formas de producción y transmisión del saber. En: Capitalismo Cognitivo propiedad intelectual y creación colectiva. Madrid: Traficantes de sueños.

LAZZARATO, MAURIZIO. (2007). La filosofía de la diferencia y el pensamiento menor. Bogotá: Universidad Central. Instituto de Estudios Culturales.

MONCAYO, VÍCTOR. (2007). Viejas y nuevas exclusiones de la educación superior colombiana. En: Debate sobre la educación superior. Serie documentos de trabajo. Bogotá: Planeta Paz.

MÚNERA, LEOPOLDO. (2007). La universidad en el siglo xxI. En: Debate sobre la educación superior. Serie documentos de trabajo. Bogotá: Planeta Paz.

RULLIANI, ENZO. (2004). El conocimiento cognitivo ¿un déjà-vu? En: Capitalismo Cognitivo propiedad intelectual y creación colectiva. Madrid: Traficantes de sueños.

VIRNO, PAOLO. (2003). Gramática de la multitud. Madrid: Traficantes de Sueños 